Revista Monografias Ambientais

Santa Maria, v. 14, n. 3, Set-Dez. 2015, p. 102-108

Revista do Centro de Ciências Naturais e Exatas - UFSM

ISSN : 22361308

\title{
Produção sustentável de alimentos em cultivo hidropônico
}

\author{
Sustainable food production in hydroponics \\ ${ }^{1}$ MENEGAES, Janine Farias, ${ }^{2}$ FILIPETTO,Jorge Eugênio, \\ ${ }^{3}$ RODRIGUES, Andrielle Magrini, 4 SANTOS, Osmar Souza dos.
}

\begin{abstract}
${ }^{1}$ Engenheira Agrônoma, Mestranda do PPGEA, Universidade Federal de Santa Maria, Santa Maria. RS, Brasil
${ }^{2} Z$ ootecnista, Colégio Politécnico, Universidade Federal de Santa Maria, Santa Maria, RS, Brasil

${ }^{3}$ Acadêmica em Agronomia, Universidade Federal de Santa Maria, Santa Maria, RS, Brasil

${ }^{4}$ Engenheiro Agrônomo, Dr., Colégio Politécnico, Universidade Federal de Santa Maria, Santa Maria, RS, Brasil
\end{abstract}

\begin{abstract}
Resumo
O cultivo hidropônico é uma forma de produção agrícola com uso de solução nutritiva em ambiente protegido, apresenta ótima produtividade por área e alta qualidade dos alimentos. Desta maneira, o trabalho teve como objetivo a produção sustentável de alimentos em cultivo hidropônico, com a utilização direta da água da chuva captada em reservatórios. Os experimentos, com alface, rúcula, tomate, physalis, nastúrcio e pastagens, foram realizados entre os anos de 2010 a 2012, no Colégio Politécnico da UFSM, (29ำ 42' S, 53ํ42' O e altitude de 95m). Conduzidos em estufa, no sistema hidropônico NFT, com utilização de água da chuva, captada e armazenada em dois reservatórios, de 7 mil litros cada. Utilizaram-se telas antiinsetos, prevenindo a entrada e o ataque dos mesmos às plantas, resultando em uma produção de baixo impacto ambiental e sem uso agrotóxico. A alta produção e qualidade dos alimentos se dão devido à proteção do ambiente com cobertura plástica, favorecendo assim o cultivo anual das olerícolas, não se detendo na sazonalidade dos alimentos. Desta forma, a hidroponia torna-se uma alternativa de produção e renda para pequenos, médios e grandes produtores, aumentando a produção de sazonal para anual, com intuito de redução e reaproveitamento de água na produção.
\end{abstract}

Palavras - chave: produção sustentável, cultivo hidropônico, problemas ambientais.

\begin{abstract}
The hydroponics is a form of agricultural production with the use of nutrient solution in a greenhouse, has excellent yield per area and high quality food. hus, the study aimed to sustainable food production in hydroponic culture, with the direct use of rainwater captured in reservoirs. The experiments with lettuce, arugula, tomato, physalis, nasturtium and pastures were conducted between the years 2010 to 2012, the Polytechnic College of UFSM, (29 42 'S, 53 42' and the altitude of 95m). Conducted in a greenhouse in NFT hydroponic system, using rain water, collected and stored in two tanks, 7 mil liters each. We used anti-insect screens, preventing the entry and the attack of the same plants, resulting in a low environmental impact production and unused pesticides. The high production and quality of food are all due to environmental protection plastic cover, thus favoring the annual crop of vegetable crops not stopping in seasonal food. Thus, hydroponics becomes an alternative production and income for small, medium and large producers, increasing the production of seasonal to annual, reduction of order and water reuse in production.
\end{abstract}

Key words: sustainable production, hydroponics, environmental problems. 


\section{Introdução}

A hidroponia é uma técnica de cultivo que visa obter produtos com excelente qualidade, sabor e aspectos externos superiores aos obtidos com agricultura tradicional, oferecendo menor risco de contaminações de doenças endêmicas. O cultivo em hidroponia é uma técnica de produção agrícola adequada às exigências de alta qualidade e produtividade com mínimo desperdício de água e nutrientes. Este sistema de cultivo vem crescendo, substancialmente, no Brasil e se apresenta como alternativa, proporcionando maior rendimento e qualidade da produção, bem como a redução da ocorrência de doenças (SANTOS et al., 2002).

O cultivo hidropônico por ser um sistema de produção sem solo e em ambiente fechado permite obter produtos de qualidade superior ao cultivo a campo. A solução nutritiva e as condições de cultivo variam para cada espécie vegetal, a qual possui diferentes potenciais produtivos e exigências nutricionais (TEIXEIRA, 1996). Assim, o ajuste químico da solução nutritiva depende da espécie, do ambiente, da época de plantio e cultivo (intensidade luminosa e temperatura) e, principalmente, da qualidade da água utilizada neta forma de cultivo (MELO; SANTOS, 2006).

Consequentemente, para que as plantas tenham bom crescimento e desenvolvimento no sistema hidropônico é necessário que haja constante equilíbrio de nutrientes na solução fornecida as plantas, permanecendo em faixas limitadas pela exigência nutricional da espécie cultivada, sem escassez nem excesso. Desta maneira, o cultivo em hidroponia necessita de um grande volume de água e, preferencialmente, de qualidade.

Em busca de um cultivo hidropônico sustentável, de menor custo financeiro e ambiental, principalmente, com a utilização de água, o objetivo desta linha de pesquisa é a reutilização da água da chuva, sendo a mesma captada em reservatórios.

A crescente busca por uma vida mais saudável tem levado a um aumento constante e irreversível no nível de exigência do consumidor em relação à qualidade salutar de sua alimentação. Há na sociedade uma relação cada vez mais forte e mais freqüente entre alimentação e saúde e o meio ambiente. E com isso, os consumidores estão exigindo alimentos livres de produtos químicos, principalmente, dos resíduos de agrotóxicos.

Entre as maneiras que se tem para obter e melhorar essas características exigidas pelo consumidor, sem afetar a produtividade, através da reutilização de água da chuva, com a adoção de técnicas de manejo da cultura que visem à redução de agrotóxicos sem afetar a qualidade e a aparência dos frutos, características estas que determinam o valor do produto, e assim satisfazem tanto o consumidor quanto o produtor.

A alface (Lactuca sativa L.) é uma planta herbácea anual, sendo considerada a hortaliça folhosa mais importante na alimentação do brasileiro, o que assegura a essa cultura, expressiva importância econômica (CARVALHO et al., 2005). Apresenta como principal vantagem o baixo valor energético, razão pela qual é frequentemente indicada na dieta alimentar, com alta demanda em saladas, planta nutracêutica (denominação a utilizada para plantas com uso nutricional e medicinal), conhecida pelas suas propriedades sedativas (SANTOS, 2000).

A rúcula (Eruca sativa L.) é uma hortaliça do tipo folhosa que tem apresentado aumento crescente de produção no Brasil, principalmente no cultivo hidropônico que possibilita oferta desta folhosa durante todo o ano. Possui rápido crescimento vegetativo, ciclo curto, porte baixo, com folhas relativamente espessas e subdivididas, o limbo tem cor 
verde-clara e as nervuras verde-arroxeadas, bordas lisas a recortadas (PURQUERIO et al., 2007). A rúcula é apreciada tradicionalmente na forma crua em salada, mas pode ser refogada, em sopas e recheio de pizzas. Apresenta sabor levemente picante semelhante aos da mostarda, é rica em proteínas, vitaminas A e C e sais minerais, principalmente cálcio e ferro.

O tomateiro (Solanum lycopersicum L.) é uma das olerícolas mais difundidas sendo consumida e cultivada em todo mundo, em diferentes sistemas de plantio e níveis de manejo cultural, originária da América do Sul. O rendimento econômico do tomateiro é resultante da combinação entre o número de frutos colhidos por unidade de área e seu tamanho individual. O tamanho de fruto para consumo "in natura" é um dos fatores de qualidade de suma importância. Assim, relacionado a produtividades dos cultivos de tomate a campo e hidropônico, tem-se valores de 118,7 t/ha/ano e 237,4 t/ha/ano, respectivamente (SANTOS, 2009).

O physalis (Physalis angulata L. e P. peruviana L.) está incorporado no quadro das pequenas frutas no Brasil, sendo o país o terceiro maior produtor, e a Colômbia o maior produtor mundial, exportando a preço médio de quinze dólares o quilo do fruto (CHAVES et al., 2005; RUFATO et al., 2008). O cultivo de physalis hidropônico vem propiciando oferta anual à demanda deste fruto, pois é uma excelente alternativa de renda para o comércio nacional e internacional, principalmente aos pequenos produtores por possuírem alto valor nutricional, sendo fonte de ferro, fósforo, vitaminas A e C, além de alcalóides e flavonóides. Suas raízes e as folhas são ricas em propriedades medicinais, utilizadas no mercado farmacológico (SANTOS et al., 2011). Já o fruto é utilizado na fabricação de geleias, sucos, compotas, sorvetes, saladas de frutas, sendo uma ótima combinação em pratos salgados ou doces (LIMA et al., 2009).

O nastúrcio (Tropaeolum majus L.) é uma planta herbácea perene, cultivada como anual, de caules prostrados, mas de hábito escandente (pode subir enrolando os pecíolos das folhas), um tanto suculenta. A planta é considerada como alimentícia de importância econômica, com ampla utilização medicinal, melífera, corante natural, hortaliça não convencional e ornamental (CESSA et al., 2009). As flores são extremamente saborosas e aperitivas, com sabor picante, devido alguns compostos sulfurados, podendo ser usadas como medicinal, alimentar e no paisagismo (CORRÊA, 1984). O hábito de uso de flores na culinária é de 140 a.C., na Idade Média as flores eram usadas para dar mais beleza aos pratos e na atualidade é prática comum na Europa, destacando-se na culinária francesa, italiana e suíça, além da Indonésia, na Ásia (MELO; SANTOS, 2006).

As forragens verdes hidropônicas (FVH) é uma tecnologia de produção de biomassa vegetal obtida através de germinação e desenvolvimento inicial das plantas a partir de sementes viáveis, de alta digestibilidade e qualidade nutricional (FAO, 2001). Desta forma, a utilização de forragem hidropônica é uma estratégia de manter o ganho de peso diário do rebanho em períodos de vazio forrageiro, principalmente para bovinocultura de leite em pequenas propriedades, assim as forragens hidropônicas vêm como um complemento as tradicionais forragem de campo nativo ou mesmo cultivado, sem competição entre os sistemas. Podem-se utilizar várias espécies tais como: cevada (Secale cereale L.), centeio (Hordeum vulgare L.), ervilhaca (Vicia sativa L.), triticale (X Triticosecale Wittmack) e outras.

Desta maneira, o objetivo do trabalho foi à produção sustentável de alimentos em cultivo hidropônico, com a utilização direta da água da chuva captada em reservatórios. 


\section{Metodologia}

Os experimentos, com as pesquisas e produções anuais de alface, rúcula, tomate, physalis, nastúrcio e pastagens, foram realizados nos anos de 2010 a 2012, no Colégio Politécnico da Universidade Federal de Santa Maria, localizado no município de Santa Maria, na região central do Rio Grande do Sul, com 29o $42^{\prime}$ de latitude sul, 53 $42^{\prime}$ de longitude oeste e uma altitude de $95 \mathrm{~m}$ acima do nível do mar.

Os experimentos foram conduzidos em estufa modelo capela com dimensões de $12 \mathrm{x}$ $21 \mathrm{~m}$ e altura de 3,0 m e orientação no sentido Norte - Sul. A estrutura de metal foi revestida com filme de policloreto de vinil (PVC) transparente de baixa densidade (150 $\mu$ de espessura), aditivado anti-UV. Com o sistema hidropônico NFT ("Nutrient Film Technique"), nas laterais da estufa o revestimento foi de telas anti-insetos, para impedir, totalmente, a entrada e o consequente ataque de insetos nas plantas, assim isentando-as de danos por pragas e, também, favorecendo a redução de doenças. A captura de água da chuva ocorreu através de calhas instaladas ao entorno das estufas, totalizando dois reservatórios, de 7 mil litros cada, para uso direto na hidroponia.

As bancadas hidropônicas foram adaptadas conforme o cultivo da espécie, para o cultivo de alface, tomate e physalis as bancadas foram formadas por três perfis hidropônicos (tubos de polipropileno) de $6 \mathrm{~m}$ de comprimento, com $10 \mathrm{~cm}$ de profundidade e espaçamento entre canais de $40 \mathrm{~cm}$. Para o cultivo de rúcula e nastúrcio utilizou-se cinco perfis hidropônicos de $6 \mathrm{~m}$ de comprimento, com $10 \mathrm{~cm}$ de profundidade e espaçamento entre canais de $20 \mathrm{~cm}$. E, para pastagens adotou-se o sistema de cultivo em caixas de $2 \mathrm{~m}$ de largura por 1,5 de comprimento, mantendo sempre uma lâmina de solução nutritiva.

As soluções nutritivas são alocadas nos reservatórios com capacidade para 500 litros cada um, acoplados a moto-bombas $0,5 \mathrm{HP}$, fornecendo uma vazão de 2,0 litros por minuto, tendo sido monitorados diariamente através das verificações de condutividade elétrica (CE) e pH. A reposição de nutrientes foi realizada através da adição de $50 \%$ das soluções quando o valor da CE sofreu diminuição de $50 \%$, ou reposição de $100 \%$ quando o valor de CE aproximava de zero. $\mathrm{O}$ pH da solução foi mantido entre 5,8 e 6,2, através da adição de ácido clorídrico $(\mathrm{HCl} 1 \mathrm{~N})$ ou hidróxido de sódio $(\mathrm{NaOH} 1 \mathrm{~N})$. Toda vez que a $\mathrm{CE}$ atingir $50 \%$ do valor inicial se fará a reposição dos nutrientes, adicionando-se metade da concentração inicial dos sais (MARTINEZ; SILVA FILHO, 2006). O controle da circulação da solução nutritiva foi feito por temporizador (timer) que acionava o conjunto durante $15 \mathrm{~min}$. com intervalos de $30 \mathrm{~min}$. no período das 6:00 às 18:00 h, e no período noturno, a cada quatro horas, permanecendo ligado durante $15 \mathrm{~min}$. O registro diário da temperatura e umidade relativa do ar foi determinado pelo termo-higrômetro digital instalado dentro da casa de vegetação.

As pesquisas e produções anuais de alface, rúcula, tomate, physalis, nastúrcio e pastagens, em cultivo hidropônico teve o intuito de avaliar as diferentes soluções nutritivas e cultivares que se adaptam ao clima e consumo da região, bem como a sua produtividade sendo viável econômica e ambientalmente. 


\section{Resultados obtidos e discussão}

A produção hidropônica por ser realizada em ambiente protegido (estufa) tem produção durante todo o ano. O cultivo em hidroponia, no sistema NFT ("Nutrient Film Technique"), é uma técnica de produção agrícola adequada às exigências de alta qualidade e produtividade com o mínimo desperdício de água e nutrientes. Este sistema de cultivo proporciona maior rendimento de massa verde e qualidade da produção, bem como a redução da ocorrência de doenças (SANTOS; DUARTE, 2009).

As soluções nutritivas constituem o ponto principal do cultivo hidropônico, uma vez que elas determinam o crescimento das plantas e a qualidade do produto final (SANTOS, 2009). Assim, para formular uma solução nutritiva o importante é considerar a cultivar, o ambiente, a época do ano (intensidade luminosa e temperatura) e, principalmente, a qualidade da água usada no cultivo hidropônico (CASTRO, 1999).

A sazonalidade da produção de folhosas, flores e frutos, ocorre devido às condições ambientais que causam danos nas plantas, o principal é o impacto das gotas de chuva nas folhas, bem como a movimentação de partículas de solo. No entanto, o cultivo hidropônico, em ambiente controlado, reduz a zero esses danos. A grande demanda de hortaliças, durante todo ano, faz com que o cultivo hidropônico ganhe espaço na preferência dos consumidores por disponibilizar o produto todo o ano e com ótima qualidade. Cada espécie foi pesquisada em um objetivo especifico, por exemplo, a alface carro chefe da produção hidropônica em todo país, visa um modelo econômico e sustentável de oferta esta folhosa o ano inteiro com menor custo. Bem como, se aplica ao cultivo de rúcula, que ultimamente adequou uma solução nutritiva $30 \%$ mais barata que a tradicional.

No que se refere aos tratamentos fitossanitários, para manejo das produções anuais de alface, rúcula, physalis, nastúrcio e pastagens não houve aplicações de defensivos agrícolas, após a instalação das telas anti-insetos, e também houve redução próxima a $30 \%$ destes tratamentos no cultivo do tomate.

A água utilizada nos experimentos hidropônicos, mais de $85 \%$ é oriunda da captação da água da chuva e armazenada em reservatórios, o que proporcionou uma economia considerável no uso de água tratada.

\section{Considerações finais}

A pesquisa visou obter formas e dados econômicos e sustentáveis para viabilizar a produção de alimentos, desta forma o cultivo hidropônico, com algumas alterações no manejo, adoção da captura da água da chuva e uso de telas anti-insetos, torna-se uma técnica rentável com disponibilidade de produto de forma continua, bem como a maior produtividade por solução nutritiva, sem grandes desperdícios de nutrientes.

\section{Referências}


CARVALHO, J. E.; ZANELLA, F.; MOTA, J. H.; LIMA, A. L. S. Cobertura morcot do solo no cultivo de alface Cv. Regina 2000, em Ji-Paraná/RO. Ciência e Agrotecnologia, Lavras, v. 29, n. 5, p. 935-939, 2005.

CASTRO, A. C. Formulación de la solución nutritiva. Parámetros de ajuste. In: MILAGROS, M.F.; GÓMEZ, I.M.C. (Edits). Cultivos sin suelo II. Curso Superior de Especialización. 2d. Almería: DGIFA-FIAPA - Caja Rural de Almería. 1999. p. 257-266.

CESSA, R. M. A.; Produção de capuchinha cultivada em vaso com diferentes doses de fósforo e potássio em casa de vegetação. Global Science Technology, n. 3, p. 1-7, 2009.

CHAVES, A.C.; SCHUCH, M.W.; ERIG, A.C. Estabelecimento e multiplicação in vitro de Physalis peruviana L. Revista Ciência e Agrotecnologia, v.29, n.6, p. 1281-1287, 2005.

CORREAA, M. P. Dicionário das plantas úteis do Brasil e das exóticas cultivadas. Rio de Janeiro: Imprensa Nacional. 1984. p. 671-673.

FAO. Manual técnico forragem verde hidropônico. Santiago, 2001. v.1, 73p.

LIMA, C.S.M.; SEVERO, J.; MANICA-BERTO, R.; SILVA, J.A.; RUFATO, L.; RUFATO, A.R. Características físico-químicas de physalis em diferentes colorações do cálice e sistemas de condução. Revista Brasileira de Fruticultura, Jaboticabal, v. 31, n. 4, p. 10601068, 2009.

MARTINEZ, H. E. P.; SILVA FILHO, J. B. Introdução ao cultivo hidropônico de plantas. 3‥ Ed. Viçosa: UFV, 2006. 111p.

MELO, E. F. R. Q.; SANTOS, O. S. Comportamento de nastúrcio (Tropaeolum majus L.) em hidroponia. Horticultura Brasileira, v.24, n.1, p. 2542-2549, 2006. (Suplemento do Congresso).

PURQUERIO, L. F. V. O mercado de rúcula. Pesquisadores Científicos. Instituto Agronômico/APTA - Centro de Horticultura, 2007. Disponível em:<http://www.iac.sp.gov.br/Tecnologias/Rucula/Rucula.htm> Acessado em: 12 jun. 2011.

RUFATO, L.; RUFATO, A.R.; SCHLEMPER, C.; LIMA, C.S.M.; KRETZSCHMAR, A,A. Aspectos técnicos da cultura da physalis. Pelotas: UFPel, 2008. 100p.

SANTOS, O. S. Hidroponia da alface. Santa Maria: Imprensa Universitária, 2000, 160p.

SANTOS, O. S.; SCHMIDT, D.; NOGUEIRA FILHO, H.; LONDERO, F. A. Cultivos sem solo - Hidroponia. 2ª reimpressão. Santa Maria: UFSM/CCR, 2002. 107p.

SANTOS, O. S; DUARTE, T. S. Cultivo hidropônico do tomateiro. In: SANTOS, O.S. Hidroponia. Santa Maria: UFSM / Colégio Politécnico, 2009. p. 289-307. 
SANTOS, O.S. Hidroponia. Santa Maria: UFSM / Colégio Politécnico, 2009. 392p.

SANTOS, O.S.; NOGUEIRA FILHO, H.; FRONZA, D.; FILIPETTO, J.E.; BASSO, D.P. Elaboração de solução nutritiva para produção de tomate com cacho único. UFSM, Colégio Politécnico, 4p. (Informe Técnico 01/2011). 2011.

TEIXEIRA, N. T. Hidroponia: uma alternativa para pequenas propriedades. Guaíba: Agropecuária, 1996. 86p. 